\title{
Sub Ångstrom imaging of dislocation core structures: How well are experiments comparable with theory?
}

\author{
C. Kisielowski ${ }^{1}$, B. Freitag ${ }^{2}$, X. Xu ${ }^{3}$, S.P Beckman ${ }^{4}$, D.C. Chrzan ${ }^{5}$ \\ ${ }^{1}$ National Center for Electron Microscopy, Material Sciences Division, Lawrence \\ Berkeley National Laboratory, One Cyclotron Rd., Berkeley CA 94720, USA
}

${ }^{2}$ FEI Company, Achtseweg Noord 5, Building AAE, 5600 KA Eindhoven/Acht, The Netherlands

${ }^{3}$ Hitachi Global Storage Technology, 5600 Cottle Rd., San Jose, CA 95193, USA

${ }^{4}$ The Institute for Computational Engineering and Sciences, University of Texas, Austin, Texas 78712, USA

${ }^{5}$ Materials Science and Engineering, University of California, Berkeley CA 94720, USA

Keywords: Dislocation cores, gold, GaAs, TEM, STEM, EELS, ab initio electronic structure total energy calculations

Corresponding author: CFKisielowski@lbl.gov 


\begin{abstract}
During the past 50 years Transmission Electron Microscopy (TEM) has evolved from an imaging tool to a quantitative method that approaches the ultimate goal of understanding the atomic structure of materials atom by atom in three dimensions both experimentally and theoretically. Today's TEM abilities are tested in the special case of a Ga terminated 30 degree partial dislocation in GaAs:Be where it is shown that a combination of highresolution phase contrast imaging, Scanning TEM, and local Electron Energy Loss Spectroscopy allows for a complete analysis of dislocation cores and associated stacking faults. We find that it is already possible to locate atom column positions with picometer precision in directly interpretable images of the projected crystal structure and that chemically different elements can already be identified together with their local electronic structure. In terms of theory, the experimental results can be quantitatively compared with $a b$ initio electronic structure total energy calculations. By combining elasticity theory methods with atomic theory an equivalent crystal volume can be addressed. Therefore, it is already feasible to merge experiments and theory on a picometer length scale. While current experiments require the utilization of different, specialized instruments it is foreseeable that the rapid improvement of electron optical elements will soon generate a next generation of microscopes with the ability to image and analyze single atoms in one instrument with deep sub Ångstrom spatial resolution and an energy resolution better than $100 \mathrm{meV}$.
\end{abstract}

\title{
Introduction
}

Often, there is an intimate link between a physical method and an object that can be investigated because of the method invention. Transmission Electron Microscopy (TEM) and dislocations are linked in this manner ever since the first images of dislocations were recorded in electron microscopes 50 years ago [1-3]. The inner material's structure became accessible revealing dislocations that could be studied and very successfully related to materials properties. TEM continues to make unique contributions to dislocation research.

Certainly, progress was gradual and our knowledge about dislocations evolved as the methods improved. For example, a resolution of a few nanometers allowed for a 
determination of Burgers vectors [4], the weak beam technique pushed TEM resolution towards one $\mathrm{nm}$ and revealed splitting of dislocations into partials [5-7], High Resolution TEM allowed for imaging of dislocation cores with near atomic resolution $(\sim 0.2 \mathrm{~nm})$ [8], kinks on individual partial dislocations could be studied in a plane view configuration by imaging with forbidden reflections [9], and Scanning TEM in combination with Electron Energy Loss Spectroscopy (EELS) accesses impurity segregation at dislocation cores and their local electronic structure [10]. Resolution in electron microscopy has reached by now sub Ångstrom values [11,12].

Nevertheless, it became also clear that even in pure materials such as $\mathrm{Si}, \mathrm{Ge}$, or GaAs individual dislocations exhibit significant deviations from average predictions. In the particular case of dislocations in semiconductors the large body of work by H. Alexander and coworkers pointed out the complexity of the problem $[13,14]$ that may relate to the interaction of dislocations with point defects [15], contributions from core energies [16], or the formation of point defects by moving dislocations [17-19]. As a result, quantitative TEM investigations of dislocations exhibit an unusual large data scatter [20] that is commonly of unknown origin.

Therefore, it is desirable to characterize individual dislocations in terms of their geometry, core structure, chemical composition, and electronic structure with truly atomic resolution and to compare the results with state-of-the-art theoretical calculations. It is timely to re-visit this long-standing problem since novel TEM abilities and progress with theoretical calculations raise hopes to finally merge theory and experiment. This contribution describes investigations that reach for this goal. Part of the described results was briefly reported in a recent letter [21].

\section{Instrumental improvements}

A visual impression of the achieved improvements of TEM imaging at atomic resolution is given in Figure 1 that shows images of dislocation cores in gold [110].

In the 1980's and 1990's lattice imaging with a resolution of about $0.18 \mathrm{~nm}$ was the most common imaging technique yielding atomic resolution, as shown left in Fig. 1. This lattice image depicts intersecting partial dislocations that interacted to generate a stair rod dislocation and associated stacking faults. It is an interferogram of scattered beams and - 
in general - image simulations are required to relate the recorded intensity pattern to the materials structure. Directly interpretable images with atomic resolution became available more recently by the reconstruction of the electron exit waves from focal series of lattice images [23, 24] (Fig. 1, center) or by recording HAADF-STEM images (Fig. 1, right). For example, it is readily recognized in Fig. 1 (center) that the recorded defect is a 1/6 [110] dislocation with a hollow core that is surrounded by a ring of 5 atomic columns, a structure that is not obvious from the single lattice image shown on the left.
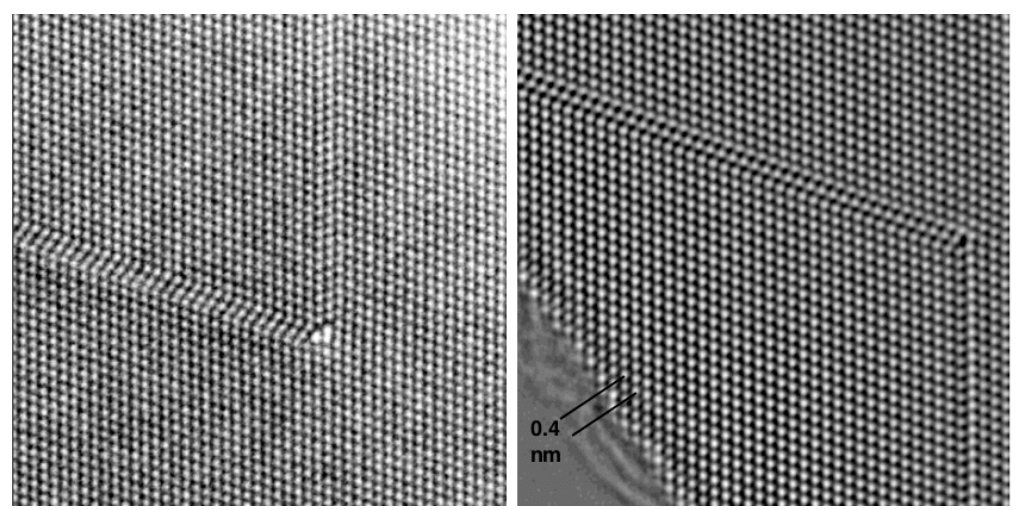

Figure 1: Gold [110] images of resolved dislocation cores recorded with different techniques. Left: High voltage lattice image recorded with the Berkeley Atomic Resolution Microscope operated at 800 kV in the 1980's. The instrument has a Scherzer point resolution of 1.7 A. (Courtesy J.M. Penisson). Center: Phase of the electron exit wave reconstructed from a focus series of 20 lattice images recorded with a Cs corrected microscope in 2002 (Juelich) operated at $200 \mathrm{kV}$ [22]. The instruments information limit was 1.4 A. Right: High Angle Annular Dark Field Image recorded with a TITAN microscope operated at $300 \mathrm{kV}$ and recorded in 2005 without a Cs corrector. In this configuration the instrument easily resolves Si [110] dumbbells $(0.136 \mathrm{~nm})$.

Single lattice images recorded in an $200-300 \mathrm{kV}$ field emission microscope through an objective lens with a finite spherical aberration coefficient exhibit even more complex intensity patterns than high voltage instruments because of rapid oscillations of their contrast transfer function [26] as shown by the simulated lattice image of a $90^{\circ}$ partial dislocation in silicon on the left in Fig. 2. Only a reconstruction of electron exit waves completely removes the image artifacts and uncovers the projected crystal structure. In fact, one can hope that even a single or double periodic core reconstruction $[16,25]$ can 
be identified (Fig. 2). Further, the resolution in reconstructed exit wave images is given by the microscopes information limit, which can exceed the Scherzer point resolution by a factor of more than 2 [26]. Therefore, sub-Ångstrom resolution can be achieved [27, $28]$ and dislocation cores in $\langle 110\rangle$ oriented semiconductors can be imaged with truly atomic resolution, which was not possible before since their dumbbell spacing in $<110>$ projection is often smaller $(<0.15 \mathrm{~nm})$ than the typical $0.18 \mathrm{~nm}$ Scherzer point resolution of traditional phase contrast microscopes [27]. Moreover, residual lens aberration can be corrected by numerical phase plates in the exit wave reconstruction process [24]. This approach complements the successful correction of lens aberrations by hardware correctors [29] and for reasons outlined in References 22 and 30 it is most advantageous to combine both methods. This combined hardware/software approach was used to generate the center image of Fig. 1.
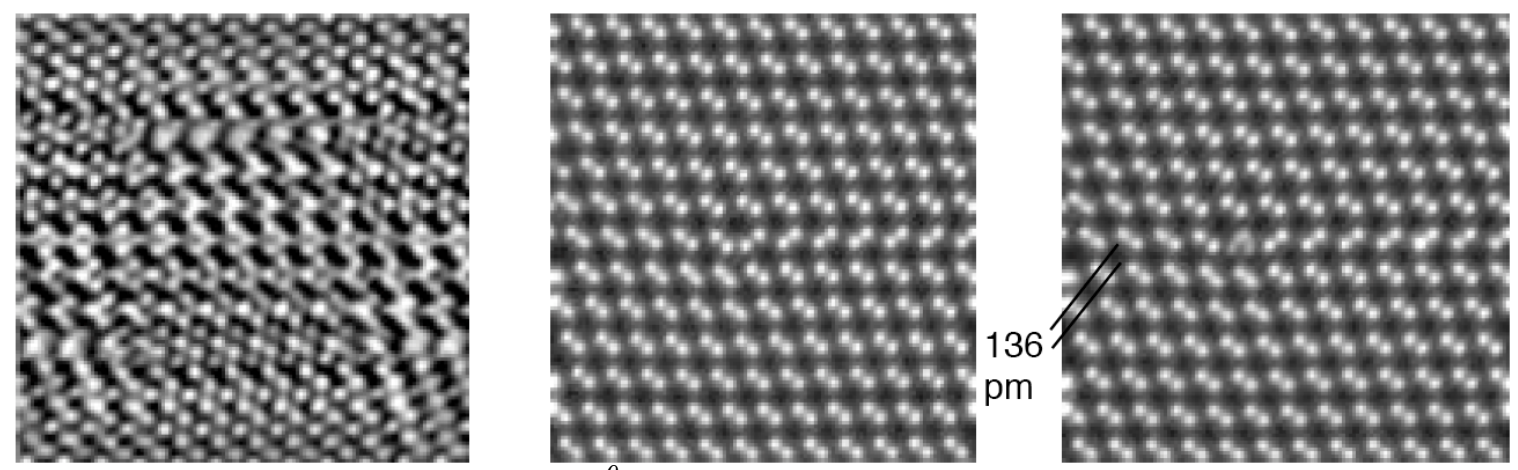

Figure 2: Simulated images of a $90^{\circ}$ partial dislocation in $\mathrm{Si}[110](300 \mathrm{kV}, \mathrm{Cs}=0.6 \mathrm{~mm}$, - $260 \mathrm{~nm}$ underfocus.

Left: Single lattice image. Center: Reconstructed phase image of a single periodic core. Right: Reconstructed phase image of a double periodic core.

The outstanding signal to noise ratio in exit wave images - if compared to other imaging techniques (Fig. 1) that provide complementary information [31] - is a consequence of bright field imaging, aberration correction, and resolution enhancement. The significance of these achievements becomes obvious if one considers the precision as to which atom column positions can be quantitatively extracted from an image. It is well established [32] that the standard deviation $\sigma$ of a column position measurement is a function of the Rayleigh resolution $\rho$ and the number of electron counts $\mathrm{N}$ in an image: $\sigma \sim \rho / \mathrm{N}^{1 / 2}$. Since 
$\rho$ is about $1 \AA$ and images can be recorded with 10000 counts, atom column positions can be determined with picometer precision if effects of lens aberrations in the image are small enough or at least constant across a region of interest. A precision of a few pm enables the detection of elastic displacement fields of line defects and even allows for the identification of point defects through their associated strain field. It is worth noting that such a precision seems beyond the current ability of theory to predict absolute column positions in a solid.

If defects are imaged with sub-Ångstrom resolution they must reside in crystals that are thinner than $10 \mathrm{~nm}$ [27]. Consequently, only a few thousand atoms are in the proximity of the defect. By careful combination of elasticity theory methods with ab initio methods, a similar volume can be treated computationally. Therefore, it has become possible to merge theory and experiment considering identical numbers of atoms and to model electron transparent sample by state-of-the-art theory. Such an approach is beneficial to both sides: theory can be used to guide the experiments and the experiments can now be exploited to improve on theoretical considerations.

\section{Computational abilities}

In the framework of linear elasticity the line energy $E$ of a dislocations can be described by a core term $E_{c}$ that is difficult to estimate but a significant contribution to the dislocation's total energy [16]. Within the isotropic limit an elastic contribution $\mathrm{E}_{\mathrm{e}}$ of an edge dislocation takes the form [33]

$$
\mathrm{E}=\mathrm{E}_{\mathrm{c}}+\mathrm{E}_{\mathrm{e}}=\mathrm{E}_{\mathrm{c}}+\mu \mathrm{b}^{2} / 4 \pi(1-v) \log \left(\mathrm{R} / \mathrm{r}_{\mathrm{c}}\right)
$$

where $b$ is the magnitude of the Burgers vector, $v$ is the Poisson ratio, $\mu$ is the shear modulus, $\mathrm{R}$ is a long range cutoff radius, and $r_{c}$ is the dislocations core radius. In the absence of computational schemes that would reliably determine core energies it was suggested to adjust $r_{c}$ such that the core energy contribution is minimal [33]. In this case, the magnitude of $r_{c}$ is typically $b / 3$ through $b / 4[16,33]$ and - in Fig. 1 (center) for

example - it is seen that it is comparable to the distance form the dislocation's 
geometrical core to the first cylinder of surrounding atom columns that form the 5 fold ring.

Therefore, one expects that the linear elasticity theory reproduces well the location of most atom column positions surrounding a dislocation core with the possible exception of the first shell(s). The arrangement of these core atoms, however, holds the key information about core energies. Indeed, Hÿtch et al. [34] recently verified this expectation and even demonstrated that the difference between anisotropic and isotropic elastic contributions can be distinguished from the displacement fields of a $1 / 2$ [110] edge dislocation in silicon recorded in a lattice image. However, considerations about column positions close to the core cutoff radius remain excluded and excluded are strain relaxation effects in thin foils.

Beckman et al. [35] recently reviewed ab initio electronic structure total energy methods and concluded that semi-periodic calculations employing capped cylinders are a viable means of studying dislocation cores in diamond cubic semiconductors. Since TEM samples are of finite thickness, a correction for elastic relaxation caused by the Eshelby twist [36] of the screw component is incorporated in the calculated atomic structure. Our recent experiments focused on isolated dislocations in GaAs [21].
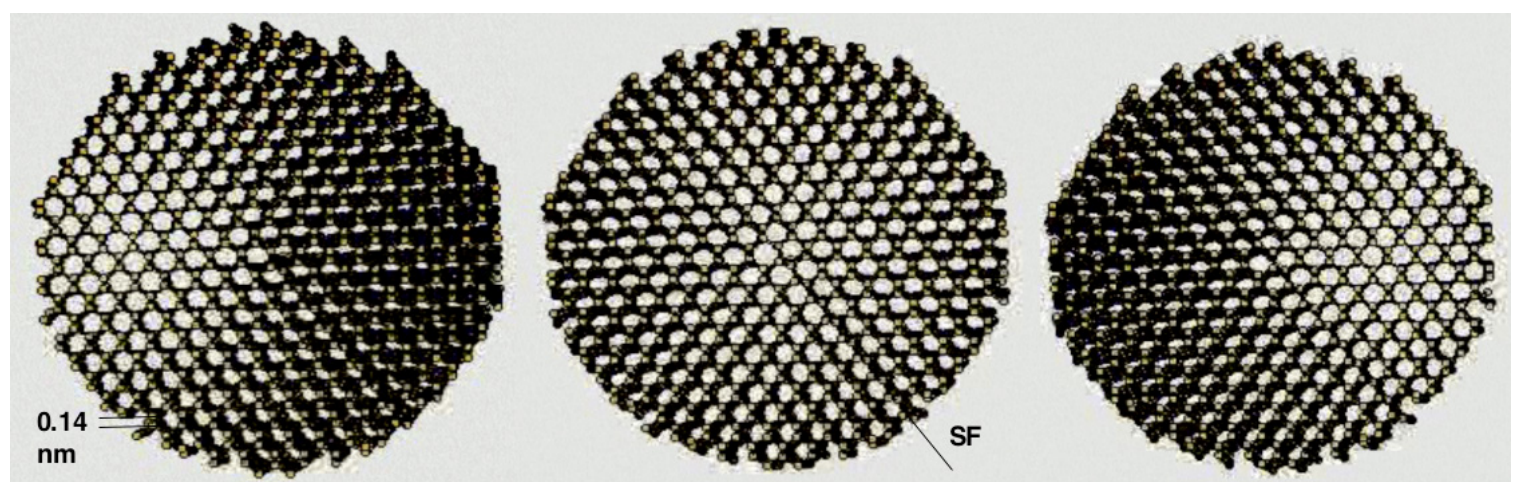

Figure 3: Calculated structure of a $30^{0} 1 / 6$ [112] partial dislocation in silicon in [011] projection showing the effect of Eshelby twist. The cylindrical crystal is $\sim 6 \mathrm{~nm}$ thick, the dislocation core is visible in the cylinder's center, and the stacking fault (SF) plane is marked. The left and right images are tilted by plus and minus 0.2 degree away from the perfect zone orientation shown in the center. 
Figure 3 shows the isotropic elasticity theory calculated structure of a $30^{\circ} 1 / 6$ [112] partial dislocation in [011] projection where strain relaxation caused by Eshelby twist is included. Three snapshots of an animation are displayed where the crystal is tilted by + 0.2 degree, across the [011] zone axis to -0.2 degree. In Figure 3 it can be seen that a clear image of atom columns is restricted to a circular window of roughly $2 \mathrm{~nm}$ diameter. Outside of this window column twist smears out the picture. This is the effect of Eshelby twist and the image series shows that it couples strongly to a moderate crystal tilt of only $\pm 0.2^{0}$ because the window moves rapidly across the field of view. Obviously this effect must be reproduced in every experiment that aims at imaging of screw dislocations.

Beyond local geometrical and compositional investigations of dislocation cores, it is of interest to understand their electronic band structure [37]. However, only recently it became possible to locally probe for low energy losses since monochromators became available [38]. Defect states in the room temperature band gap of GaN (3.3 eV) and InN $(1.7 \mathrm{eV})$ were recently observed by low loss EELS [39, 40] with nm spatial resolution. Currently signal to noise levels hamper the determination of a dislocation's electronic structure although such experiments have been attempted [41].

\section{Merging experiments with theory}

A reconstructed phase image and a HAADF-STEM image of a $30^{\circ} \mathrm{Ga}$ terminated partial dislocation in GaAs:Be and the associated stacking faults are shown in Figure 4. We take advantage of the large image contrast and small noise levels in reconstructed electron exit wave images to extract column positions. Similarly, we exploit the strength of STEM to simultaneously record an image while stepping a $0.1 \mathrm{~nm}$ probe across an area of interest to record EELS spectra [21].

The information about column positions in the HREM image was processed to produce a histogram for the $0.14 \mathrm{~nm}$ Ga-As dumbbell spacing that is shown as an inset in Figure 4. From the repetitive measurements we find that the Ga-As dumbbell spacing can be reproduced with an error of only $\sigma=2 \mathrm{pm}$ in the bulk of the crystal. Unexpectedly, this distance appears elongated by up to $12 \%$ if it is measured along the stacking fault. The same effect is present in the HAADF STEM image. It is less apparent there because of larger uncertainties that arise from the higher noise levels in unfiltered HAADF-STEM 
images (Figure 1). On the other hand core loss EELS measurements uncover a possible reason for the projected bond length elongation since we detect higher $\mathrm{Ga}$ and $\mathrm{Be}$ spectrum signal at the stacking fault (Inset Figure 4) relative to the As and $\mathrm{C}$ reference signals that are reasonably constant. Careful investigation of the structure predicted by $\mathrm{ab}$ initio methods confirms that the EELS results may be due to double segregation of $\mathrm{Ga}_{\mathrm{As}}$ and $\mathrm{Be} \mathrm{Ga}_{\mathrm{Ga}}$, although such effects are not intuitive [21].

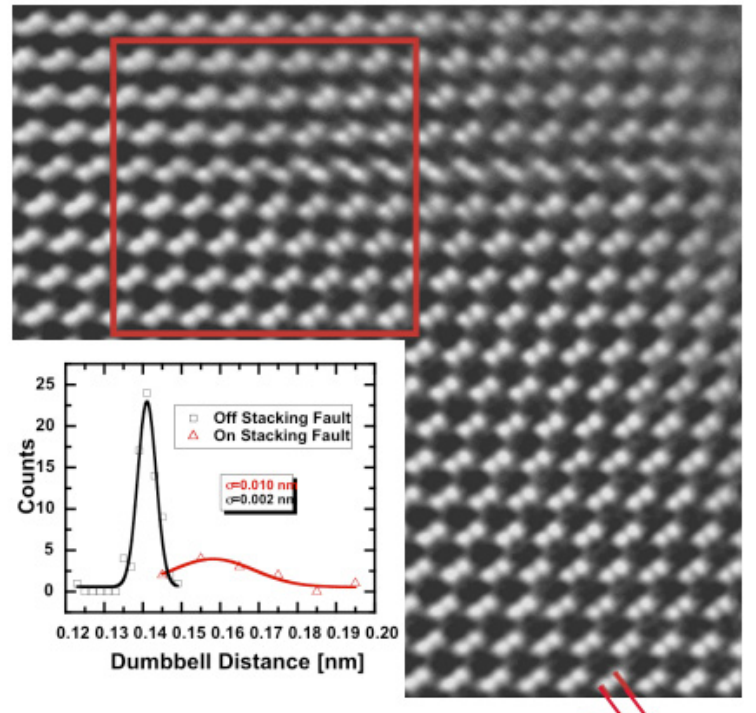

$0.14 \mathrm{~nm}$

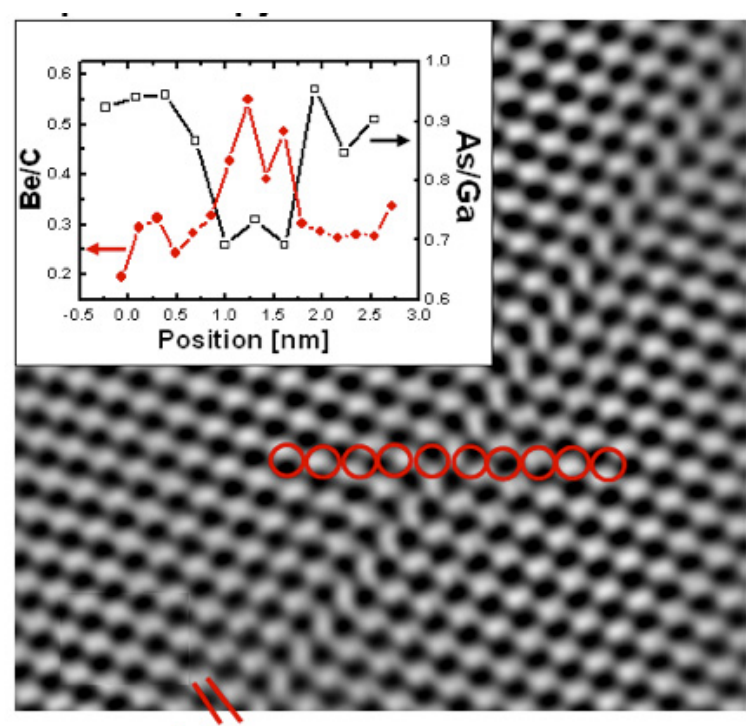

$0.14 \mathrm{~nm}$

Figure 4

Left: Phase of the electron exit wave reconstructed from 20 lattice images recorded in the Berkeley OAM microscope that provides $0.08 \mathrm{~nm}$ of resolution. Inset: Histogram of dumbbell spacing from the bulk of the material and from the stacking fault.

Right: Noise filtered HAADF-STEM image of a stacking fault recorded with a $0.1 \mathrm{~nm}$ probe in the Super STEM / UK. Circles mark positions were EELS spectra from C, Ga, As, and Be edges were recorded. The inset shows the spatial fluctuation of these elements across the stacking fault revealing Ga and Be segregation [21].

The noise levels in both images of Figure 4 prohibit an identification of As or Ga atoms from intensity measurements of one dumbbell. Existing intensity differences between the two maxima are simply overwhelmed by noise because of the element's similar atomic numbers $\left(Z_{\mathrm{Ga}}=31, \mathrm{Z}_{\mathrm{As}}=33\right)$. Therefore, we consider the change of dumbbell intensity ratios with crystal thickness, which is Z-dependent [26]. A comparison of this ratio with 
image simulations allows for a unique assignment of one element to one of the face centered cubic sub-lattices of the zincblende structure as shown in Figure 5. The procedure reveals a Ga termination of the imaged dislocation core.

The $a b$ initio calculated models provide input parameters for multislice calculations of lattice images that are subsequently reconstructed to produce phase images of the electron exit wave, which can be compared with the experiment. The phase images of Figure 5 show results of such simulations that are executed assuming a constant magnitude of azimuthal crystal tilt of $0.2^{0}$ and rotating the tilt axes in the image plane in steps of $90^{\circ}$. We find that the simulation labeled $320^{\circ} / 0.2$ (in plane angle/out of plane angle) matches the experiment best and it is clear that Eshelby twist must be considered to reproduce the experiment.
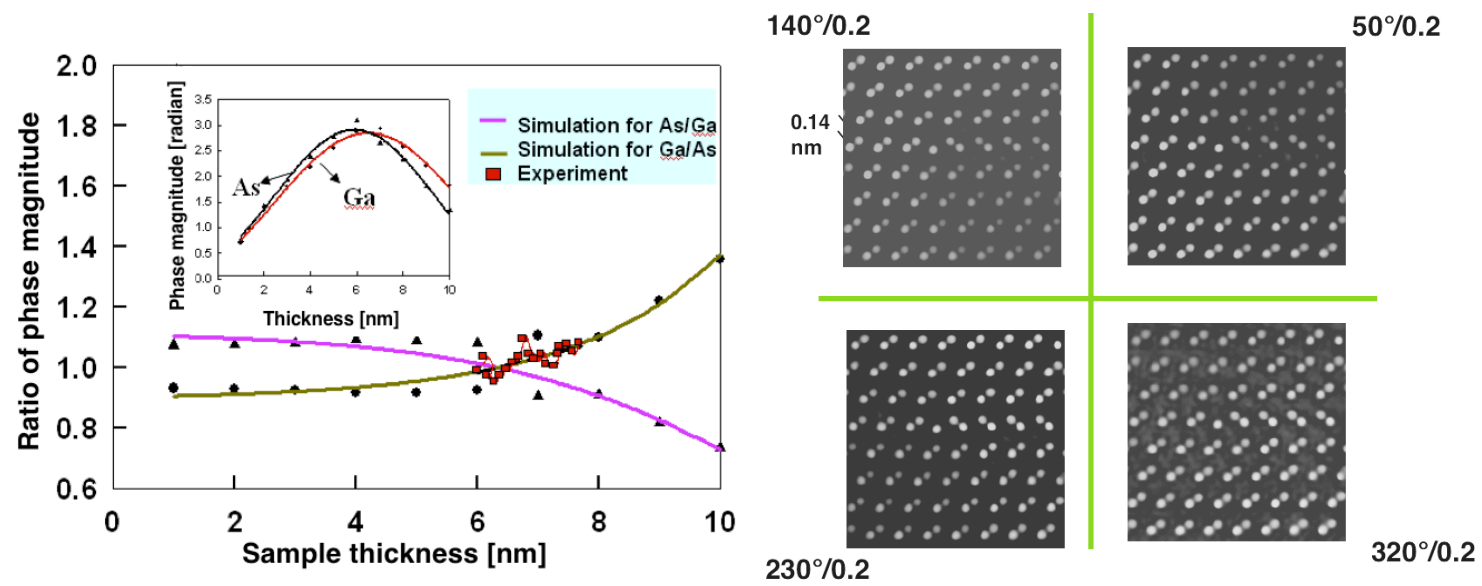

Figure 5

Left: Determination of the occupancy of the 2 fcc sub-lattices in the zinkblende structure with As and Ga atoms by comparing thickness dependent intensity measurements with results from multislice calculations [42].

Right: Effect of the Esheby twist on the intensity distribution around a $30^{0}$ Ga terminated partial dislocation in GaAs in case of four different crystal orientations as indicated.

The final step towards a quantitative comparison between experiments and theory consists of recovering atom column positions from the intensity maxima of simulated and recorded phase images and of minimizing the difference between equivalent column positions by a least square method across the area of interest. Residuals are represented in 
form of a vector plot where the vector's length' and directions quantify the displacement between a calculated and measured atom column position (Figure $6 \mathrm{a}, \mathrm{b}$ ).

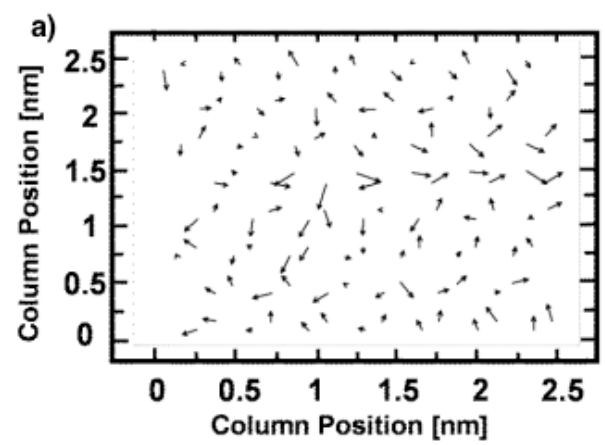

c)
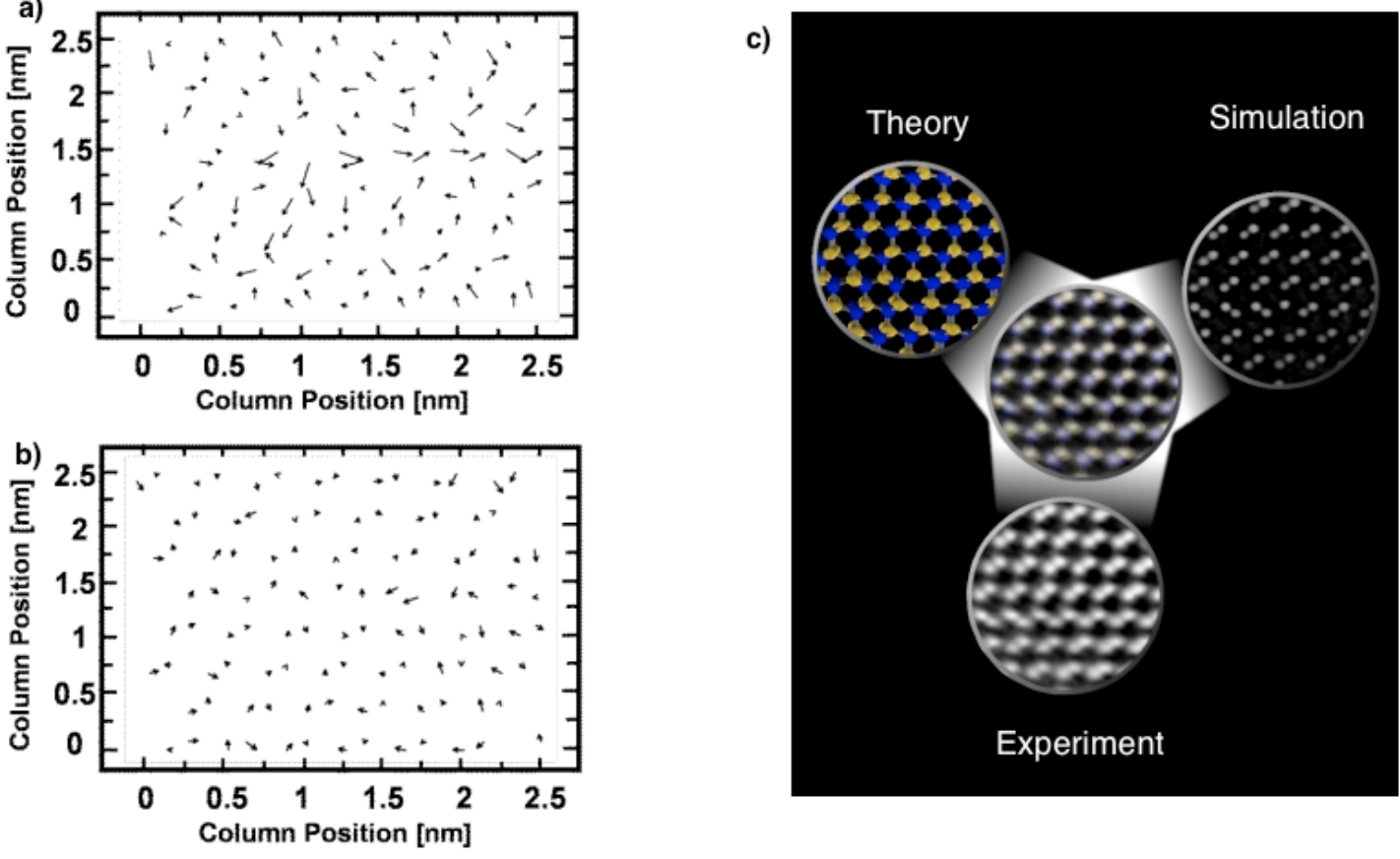

Figure 6

a), b) Vector fields describing local deviations between calculated and measured atom column positions. a) compares theoretical expectations for an ideal dislocation with the experiment; the magnitude of the largest vectors is $18 \mathrm{pm}$. b) compares a model with the experiment after manual correction to atomic positions is incorporated; the magnitude of the largest vectors is $10 \mathrm{pm}$. c) symbolizes the unity of theory, image simulations, and experiment.

Figure 6 a) proves that the theoretical description of an ideal dislocation embedded in a simulated electron transparent crystal agrees remarkably well with the corresponding experiment: In most part of the displayed area the residual deviations remain below 10 $\mathrm{pm}$. Only close to the dislocation's core and along the stacking fault they reach at most $18 \mathrm{pm}$. This is not surprising since impurity segregation was observed. It is possible to determine the atomic column positions to better than $10 \mathrm{pm}$ everywhere if experimentally guided correction are added to the atomic columns shown in Figure 6 b). Thereby, we show that theory, simulations, and experiments can be merged to obtain residual 
discrepancies that are smaller than $10 \mathrm{pm}$ over a $6.25 \mathrm{~nm}^{2}$ large crystal area in a $\sim 6 \mathrm{~nm}$ thick relaxed sample as symbolized in Figure $6 \mathrm{c}$ ).

\section{Conclusions}

In the past 50 years phase contrast electron microscopy has uniquely contributed to our understanding of materials properties. By now the method's resolution has broken the 0.1 $\mathrm{nm}$ barrier and it is possible to combine theory and experiment to accurately identify atomic column positions with better than $10 \mathrm{pm}$ precision. The versatility of electron microscopy was broadened to include scanning electron microscopy and electron spectroscopy. Nevertheless, the zenith of these methodical developments has yet to come.
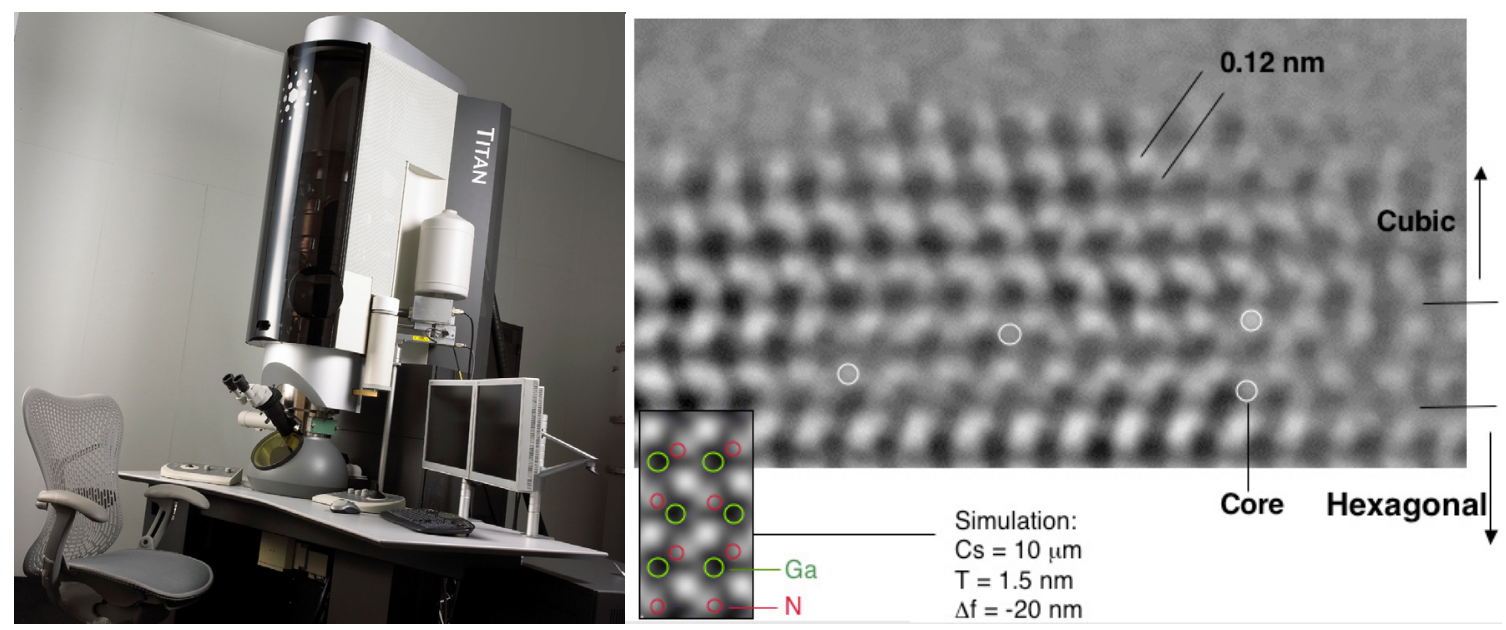

Figure 7

Left: Advanced electron optical elements will be integrated into a TITAN electron microscope [43] including spherical aberration correctors, a monochromator, and a combined spherical and chromatic aberration corrector that is currently developed by the TEAM Project [44]. Technological challenges increase rapidly the more components are involved.

Right: Single lattice image of a pair of split partial dislocations GaN recorded in a TITAN column equipped with an objective lens Cs corrector. The core of partial dislocations is resolved. The instrument already transfers information to $0.07 \mathrm{~nm}$ with an objective lens that has a pole gap of $5 \mathrm{~mm}$.

Currently, worldwide efforts are made to develop and include more advanced electron optical components into an electron microscope such as Cs correctors [29, 45], 
monochromators [38], and even Cc correctors [44]. Clearly, substantial technological challenges must still be met since the design and the stability of such instruments become increasingly demanding. The next instrument generation aims at an integration of STEM, TEM, and spectroscopy with $0.05 \mathrm{~nm}$ of spatial resolution and $100 \mathrm{meV}$ of energy resolution [44]. Platforms of such instruments are currently being tested.

These developments are science driven and it is essential to understand the potential benefit. One obvious reason for such efforts can be deduced from the content of this paper: It is desirable to investigate one and the same object with all the described tools. This aspect is addressed best by integration of all techniques into one column [46]. Further, it is quite common to praise resolution achievements. However, it is uncertain at this point if a further resolution enhancement will enable new science [44]. Surely such arguments are honorable but not necessarily strong enough to justify the huge investments. In this respect it seems most important to highlight the achievements in terms of improved signal to noise ratios and detection limits: Electron microscopy strives for the detection of single atoms of most elements from the Periodic Table [47]. Ultimately, this ability can remove the long-standing uncertainties that come from the projection of a three dimensional object into a two dimensional image. Accounting for every atom in three dimensions both theoretically and experimentally is a "holy grail" for materials sciences; if reached it offers numerous novel applications.

\section{Acknowledgements}

The authors acknowledge support of the National Center for Electron Microscopy, Lawrence Berkeley Lab, which is supported by the U.S. Department of Energy under Contract \# DE-AC02-05CH11231.

\section{References}

[1] W. Bollman, Physical Review 103, 1956, 1588

[2] P.B. Hirsch, R.W. Horne, M.J. Whelan, Philosophical Magazine 1, 1956, 677

[3] J.W. Menter, Proceedings of the Royal Society of London Series A Mathematical \& Physical Sciences 236, 1956, 119 
[4] P.B. Hirsch, A. Howie, M.J. Whelan, Philosophical Transactions of the Royal Society of London. Series A Mathematical and Physical Sciences, 252, 1960. 499

[5] I.L.F. Ray, D.J.H. Cockayne, Philosophical Magazine 22, 1970, 853

[6] H. Gottschalk, G. Patzer, H. Alexander, Physica Status Solidi A, 45, 1978, 207

[7] H. Foll, C.B. Carter, M. Wilken, Physica Status Solidi A, 58, 1980, 393-407

[8] A. Olsen, J.H.C. Spence, Phil. Mag. 43, 1981, 945

[9] H. Alexander, H.R. Kolar, J.H.C. Spence, Physica Status Solidi A, 171,1999, 5

[10] I. Arslan, A. Bleloch, E.A. Stach, N.D. Browning, Physical Review Letters 94, 2005, 025504

[11] P.D. Nellist, S.J. Pennycook, Phys. Rev. Letters 81,1998, 4156.

[12] C. Kisielowski, A. Schwartzmann, C.E. Nelson, C. Song, R. Kilaas, A. Thust, Microsc. Microanal. 6, Suppl. 2, 2000, 14

[13] H. Alexander, in Dislocation in Solids, edited by F. R. N. Nabarro (North-Holland, Amsterdam, 1986), Vol. 7, p. 113

[14] H. Alexander and H. Teichler, Dislocations in Handbook of Semiconductor Technology, edited by K. A. Jackson and W. Schroeter (Wiley-VCH, Berlin, 2000), p. 293

[15] A. Antonelli, J. F. Justo and A. Fazzio, Phys. Rev. B 60, 1999, 4711

[16] X. Blase, Karin Lin, A. Canning, S. G. Louie, and D. C. Chrzan, Phys. Rev. Lett. 84, 2000,5780

[17] E.R. Weber, Solid State Communications 60, 1986, 871

[18] C. Kisielowski-Kemmerich, H. Alexander, Fizika Tverdogo Tela, 31, 1989, 254

[19] J. Palm, C. Kisielowski-Kemmerich, H. Alexander, Applied Physics Letters 58, 1991,68

[20] H. Gottschalk, N. Hiller, S. Sauerland, P. Specht, H. Alexander, Physica Status Solidi A, 138, 1993, 547

[21] X. Xu, S.P Beckman, S. Specht, E.R. Weber, D.C. Chrzan, R.P. Erni, I. Arslan, N.D. Browning, A. Bleloch, C. Kisielowski, Physical Review Letters 95, 2005, 145501 [22] J. R. Jinschek, C. Kisielowski, T. Radetic, U. Dahmen, M. Lentzen, A. Thust, K. Urban, Mater. Res. Soc. Symposium Proceedings 727, 2002, 3 
[23] W.M.J. Coene, A. Thust, M. Op de Beeck, D. Van Dyck, Ultramicroscopy 64, 1996, 167

[24] A. Thust, W.M.J. Coene, M. Op de Beeck, D. Van Dyck, Ultramicroscopy 64, 1996, 211

[25] J. Bennetto, R.W. Nunes, D. Vanderbilt, Phys. Rev. Lett. 79, 1997, 245

[26] D.B. Williams, C.B. Carter, Transmission Electron Microscopy, Plenum Press, New York, 1996

[27] C. Kisielowski, C.J.D. Hetherington, Y.C. Wang, R. Kilaas, M.A. O'Keefe, A. Thust, Ultramicroscopy 89, 2001, 243

[28] M.A. O'Keefe, C.J.D. Hetherington, Y.C. Wang, E.C. Nelson, J.H. Turner, C.

Kisielowski, J.-O. Malm, R. Mueller, J. Ringnalda, M. Pam, A. Thust, Ultramicroscopy 89, 2001, 215

[29] M. Haider, H. Rose, S. Uhlemann, E. Schwan B. Kabius, K. Urban, Ultramicroscopy 75, 1998, 53

[30] K. Tillmann, A. Thust, and K. Urban, Microsc. Microanal. 10, 2004, 185

[31] J. Ayache, C. Kisielowski, R. Kilaas, G. Passerieux, S. Latrigue-Korinek, J. Mat. Science 40, 2005, 3091

[32] D. Van Dyck, S. Van Aert, A.J. den Dekker, A. van den Bos, Ultramicroscopy 98, 2003, 27

[33] J. P. Hirth and J. Lothe, Theory of Dislocations, Wiley, New York, 1982

[34] M. J. Hÿtch, J.-L. Putaux, J.M. Penisson, Nature 423, 2003, 270

[35] S. P. Beckman, X. Xu, P. Specht, E. R. Weber, C. Kisielowski, D.C. Chrzan, J. Phys.: Condens. Matter 14, 2002, 12673

[36] J. D. Eshelby and A. N. Stroh, Phil. Mag. 42, 1951, 1401

[37] S.P. Beckman, D.C. Chrzan, Physica B 340-342, 2003, 1001

[38] D.S. Su, H.W. Zandbergen, P.C. Tiemeijer, G. Kothleitner, M. Haevecker, C.

Hebert, A. Knop-Gerick, B.H. Freitag, F. Hofer, R. Schloegl, Micron 34, 2003, 235

[39] P. Specht, J.C. Ho, X. Xu, R. Armitage, E.R. Weber, R. Erni, C. Kisielowski, solid state comm. 135 (2005) 340.

[40] P. Specht, X. Xu, R. Armitage, E.R. Weber, R. Erni, C. Kisielowski, Physica, 2005, in press 
[41] P.E. Batson, Phys. Rev. Lett. 83, 1999, 4409

[42] McTempas simulation software, roar@totalresolution.com

[43] S. Kujawa, B. Freitag, D. Hubert, MICROSCOPY TODAY, July 2005, 16

[44] http://ncem.lbl.gov/team3.htm

[45] O.L. Krivanek, N. Dellby, A.R. Lupini, Ultramicroscopy 78, 1999, 1

[46] G. Mobus, J.M. Titchmarsh, J.L. Hutchison, C.J. Hetherington, R.C. Doole, D.J.H. Cockayne, Electron Microscopy and Analysis 2001. Proceedings. IOP Publishing. 2001, 27

[47] C. Kisielowski, J.R. Jinschek, J.R. in "Physik Mikrostruktuierter Halbleiter" 27, P. Specht, T.R. Wetherford, P. Kiesel, T. Marek, S. Malzer (eds.) Erlangen-Nuernberg, 2002, 137 